\title{
The Relationship between Gestational Diabetes and Risk Factors in Pregnant Women in Hamadan
}

\author{
Shiva Borzouei (MD) ${ }^{1, *}$, Soghra Rabiei (MD) ${ }^{2}$, Farzaneh Esna Ashari (MD) ${ }^{3}$, Zohreh \\ Zareeighane (MSc) ${ }^{4}$, Mansooreh Biglari (MD) ${ }^{5}$
}

${ }^{1}$ Departrment of Endocrinology, Faculty of Medicine, Clinical Research Development Unit of Shahid Beheshti Hospital, Hamadan University of Medical Sciences, Hamadan, Iran

2 Professor of Obstetrics and Gynecology, University of Medical Sciences, Hamadan, Iran

${ }^{3}$ Associate Professor of Social Medicine, Hamadan University of Medical Sciences, Hamadan, Iran

${ }^{4}$ Master of Science (MSc), Immunology Department, Hamadan University of Medical Sciences, Hamadan, Iran

${ }^{5}$ General physician, Hamadan University of Medical Sciences, Hamadan, Iran

* Corresponding Author: Shiva Borzouei, Assistant Professor, Departrment of Endocrinology, Faculty of Medicine, Clinical Research Development Unit of Shahid Beheshti Hospital, Hamadan University of Medical Sciences, Hamadan, Iran. Email: borzoueishiva@umsha.ac.ir

\begin{tabular}{|c|c|}
\hline \multirow{3}{*}{$\begin{array}{l}\text { Received: } 13 / 03 / 2018 \\
\text { Accepted: } 08 / 10 / 2018\end{array}$} & Abstract \\
\hline & \multirow{13}{*}{$\begin{array}{l}\text { Background and Objective: Gestational diabetes is a health problem and } \\
\text { is one of the most commonly occurring complications of pregnancy } \\
\text { worldwide, which has many effects on the mother and the fetus. The } \\
\text { present study was conducted to determine the prevalence of gestational } \\
\text { diabetes and its risk factors in Hamadan city, Iran. } \\
\text { Materials and Methods: In this cross-sectional study, the study population } \\
\text { was } 534 \text { pregnant women who were referred to control pregnancy to } \\
\text { Hamadan health center from April } 2014 \text { to December } 2016 \text {. } \\
\text { Results: The study participants were } 534 \text { with the age range of } 15-42 \text { years } \\
\text { old with an average age of } 26.8 \text { and a standard deviation of } 5.56 \text {. The } \\
\text { gestational age was } 4 \text { to } 29 \text { weeks with an average of } 8.32 \text { weeks with a } \\
\text { standard deviation of } 2.68 \text {. Of the } 534 \text { participants in this study, } 211 \\
\text { (39.5\%) had gestational diabetes and there was a significant relationship } \\
\text { between factors such as macrosomia and history of impaired glucose } \\
\text { tolerance with the prevalence of gestational diabetes. } \\
\text { Conclusion: Gestational diabetes has a relatively high prevalence in } \\
\text { Hamadan, further information is needed in this area as well as studies } \\
\text { based on new guidelines in different parts of the country. }\end{array}$} \\
\hline & \\
\hline \multirow{12}{*}{$\begin{array}{l}\text { How to Cite this Article: } \\
\text { Borzouei S, Rabiei S, Esna } \\
\text { Ashari F, Zareeighane Z, Biglari } \\
\text { M. The Relationship between } \\
\text { Gestational Diabetes and Risk } \\
\text { Factors in Pregnant Women in } \\
\text { Hamadan. Pajouhan Scientific } \\
\text { Journal. 2018; 17(1): 30-36. } \\
\text { DOI: } 10.29252 / \text { psj.17.1.30 }\end{array}$} & \\
\hline & \\
\hline & \\
\hline & \\
\hline & \\
\hline & \\
\hline & \\
\hline & \\
\hline & \\
\hline & \\
\hline & \\
\hline & Keywords: Gestational Diabetes; Prevalence; Screening \\
\hline
\end{tabular}

Copyright (C) 2018 Pajouhan Scientific Journal. This is an open-access article distributed under the terms of the Creative Commons Attribution-NonCommercial 4.0 International License (http://creativecommons.org/licenses/by-hc/4.0/) which permits copy and redistribute the material just in noncommercial usages, provided the original work is properly cite. 
بروسى رابطه ديابت باردارى با عوامل خطرساز در خانمهاى بار فار شهر هملان

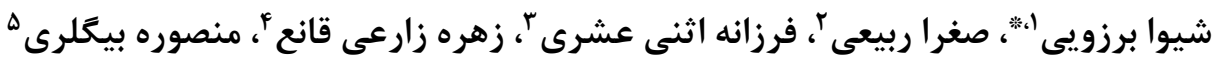
ا گروه بيمارى هاى اندوكرينولوزى، دانشكده يزشكى، واحد توسعه تحقيقات بالينى بيمارستان شهيد بهشتى، دانشكاه علوم يزشكى همدان، همدان، ايران

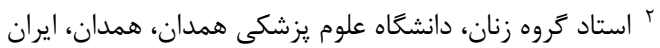

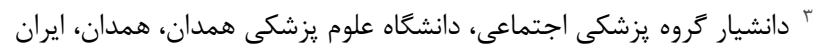

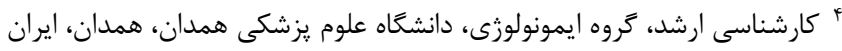

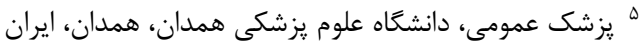

* نويسنده مسئول: شيوا برزويى، استاديار، كروه بيمارى هاى غدد، دانشكده يزشكى، واحد توسعه تحقيقات بالينى بيمارستان شهيد بهشتى، دانشكاه

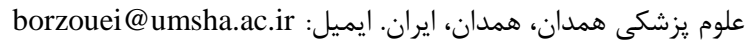

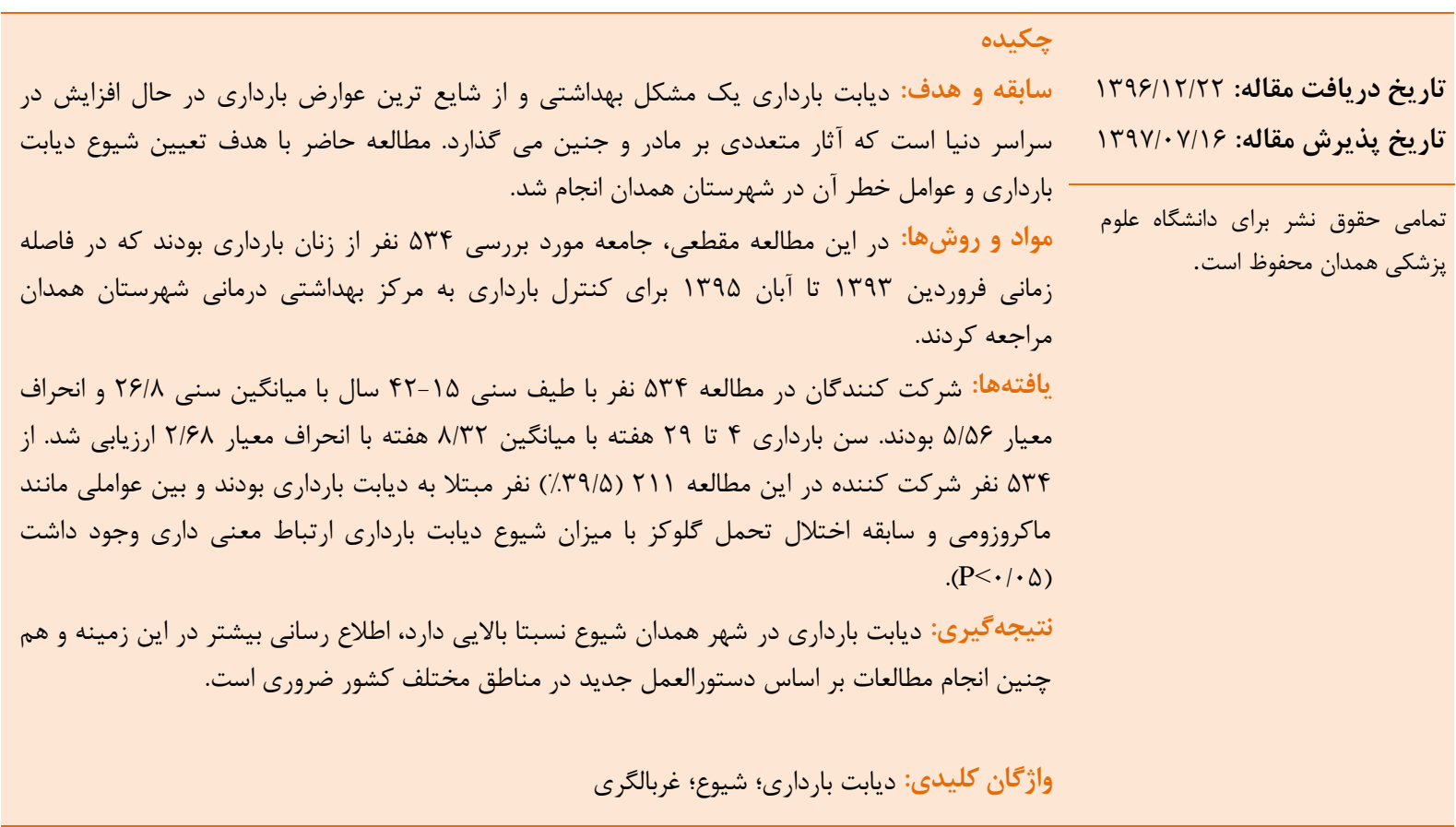

معضلات بهداشتى مطرح مى باشد، به طورى كه انتظار مىرود

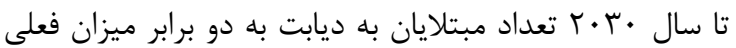

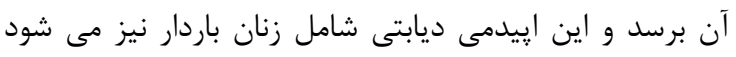

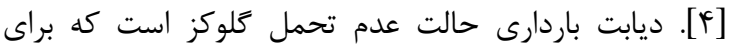

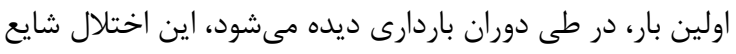

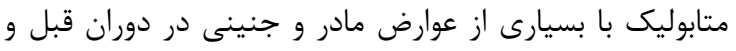

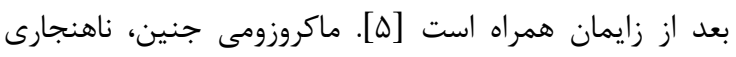
قلبى، صدمات حين زايمان مثل شكستخى هاى استخوانى - در إنى

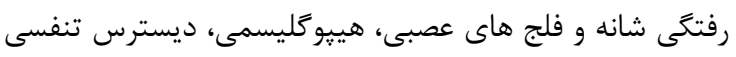

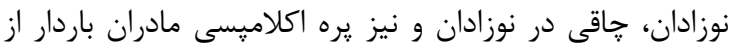

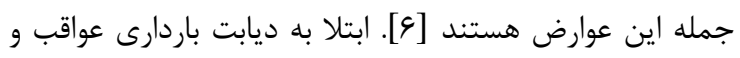

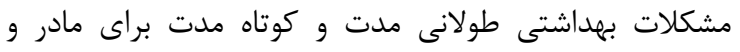
فرزند او ايجاد مى كند [V]
باردارى با تغييرات آناتوميك و فيزيولوزيك بسيارى همراه

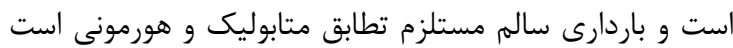

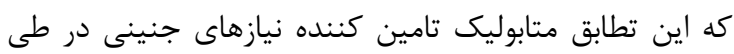

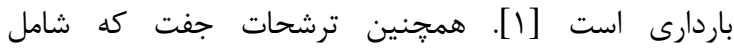
يروزسترون، لاكتوزن جفتى، هورمون آزاد كننده كورتيكوترويين

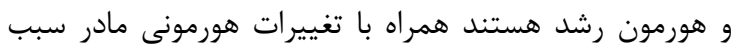

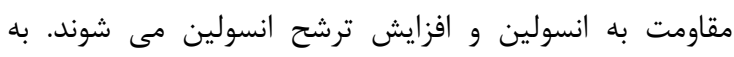

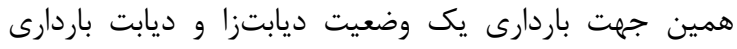

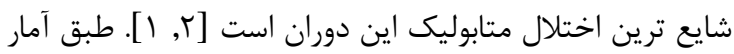

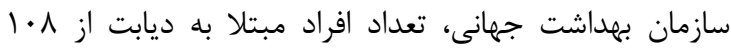

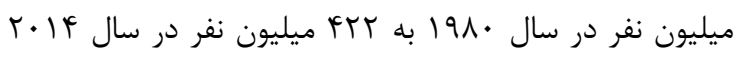

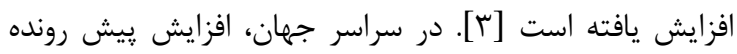

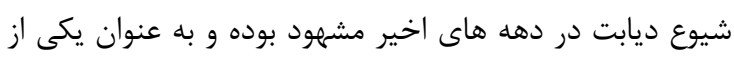




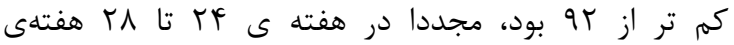

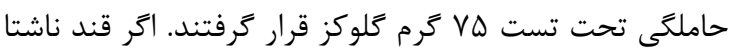

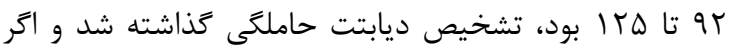
قند צrا و بالاتر بود يك بار ديخر تست تكرار و در صورت دابت

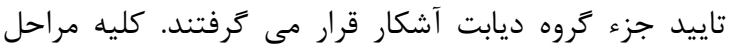
انجام كار در يك مركز بهداشت و و آزمايشعاه مربوطه انجام إندام

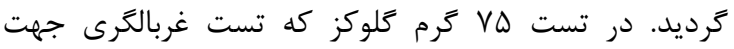

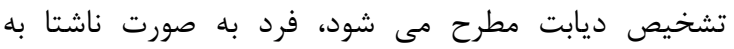

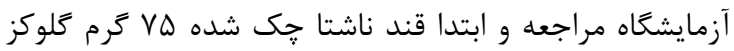

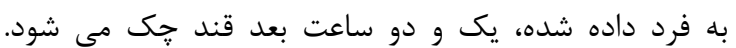

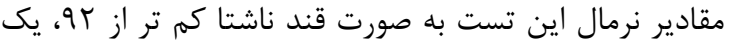

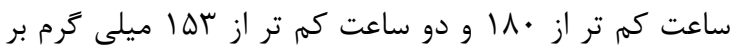

دسى ليتر مى باشد [1 إ].

در اين بررسى خانمهاى باردار با سابقه ديابت آشكار

شناخته شده و تحت درمان با داروهاى خوراكى ضد ديابت يا بردي

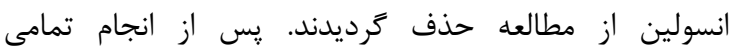

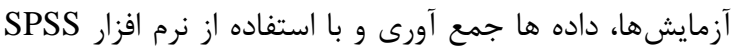

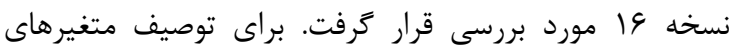

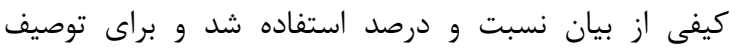
متغيرهاى كمى از ميانگين و انحراف معيار استفاده كرديد.

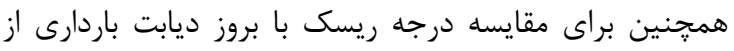

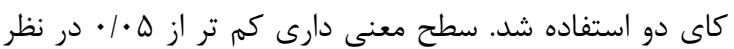
كرفته شد.

1. 1. 1)

در اين مطالعه F ماه زن باردار وارد مطالعه شدند. ميانخين

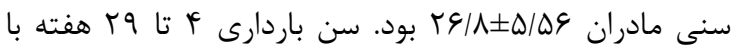
ميانگين و انحراف معيار مادران

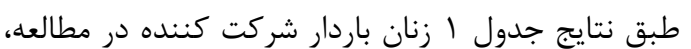

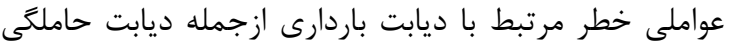

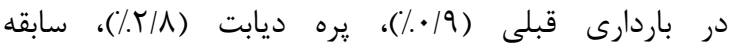

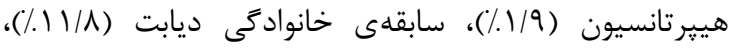

جدول ا: توزيع فراوانى شركت كنندكان بر اساس عوامل خطر

\begin{tabular}{|c|c|c|}
\hline درصد & تعداد & عوامل خطر \\
\hline .19 & $\Delta$ & ديابت حاملَّى در باردارى قبلى \\
\hline$T / \Lambda$ & 10 & يره ديابت \\
\hline $1 / 9$ & 1 . & سابقه هيير تانسيون \\
\hline $11 / 1$ & r & سابقه خانوادگى ديابت \\
\hline $1 / \pi$ & V & 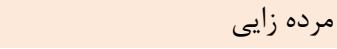 \\
\hline$\cdot / \mathrm{V}$ & r & سابقه ماكروزومى \\
\hline$\cdot / 4$ & 1 & آنومالى \\
\hline$r / T$ & ir & سابقه يره اكلاميسى \\
\hline $19 / V$ & 19 & سقط \\
\hline
\end{tabular}

شيوع ديابت باردارى در آمريكا ا تا f أ درصد كزارش شده است كه بر اساس سن، نزاد، سابقه خانوادكى ديابت، باردارى درئ

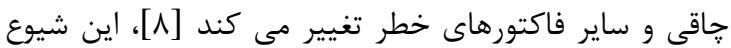

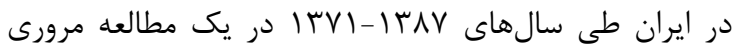

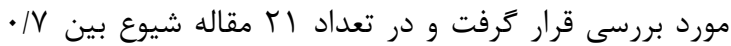

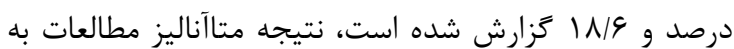

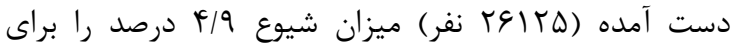

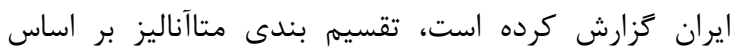
مناطق مختلف كشور نشان داد كه مناطق تهران- مركز

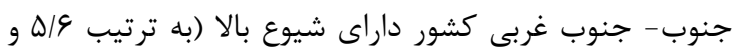

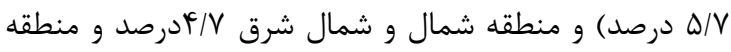

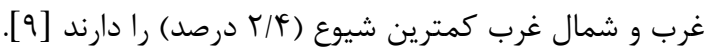

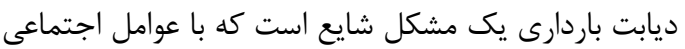
سلامت در ارتباط است. لذا اتخاذ مداخلات مناسب ماردي مانند،

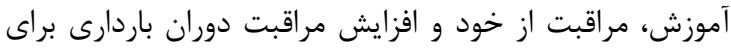

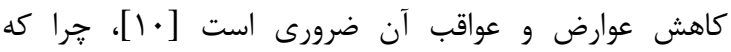
تشخيص ديابت باردارى بسيار مهمم است و با درمان صروان (شامل

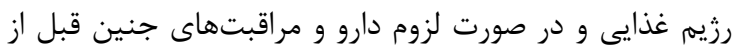

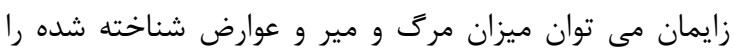

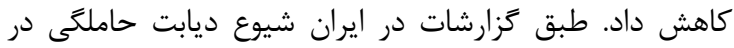

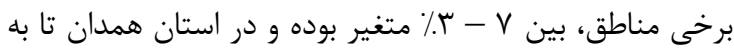
حال شيوع اين بيمارى و عوامل خطر مطالعه نشده است. با انجام

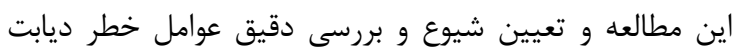

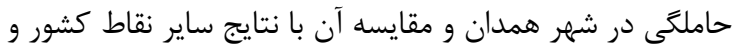

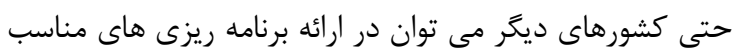

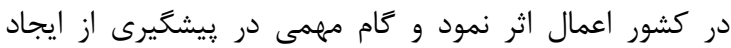

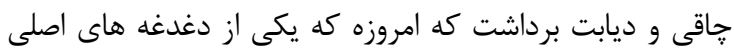

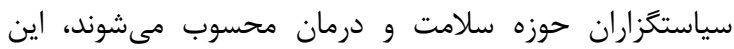
مطالعه با هدف تعيين شيوع ديابت باردارى و تعيين برخي

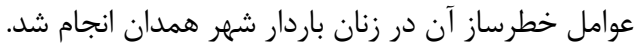

مواد و روشها

مطالعه توصيفى-تحليلى و از نوع مقطعى بود. جامعه مورد

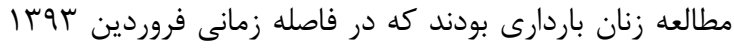

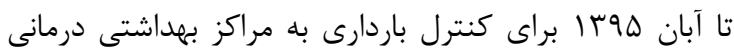

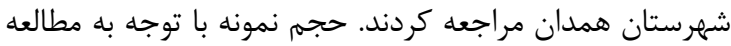

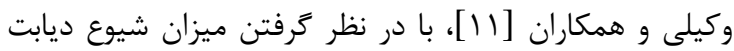

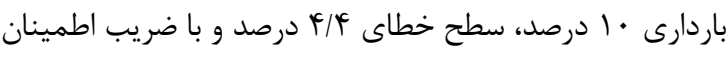

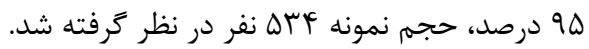

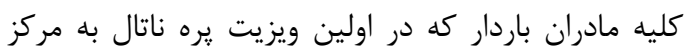

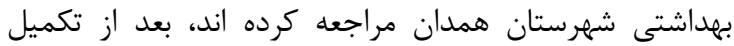

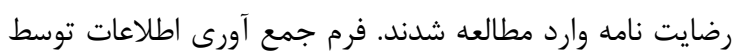

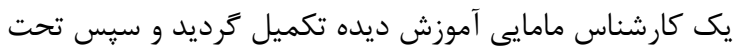

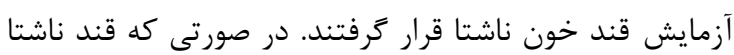


باردارى و 9/ • درصد ديابت آشكار داشتند. ب درصد نيز همراه با

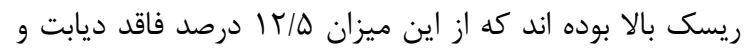

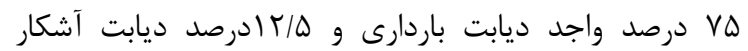
داشتند.

با توجه به جدول r، ارتباط معنى دارى بين شيوع ديابت

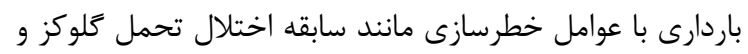

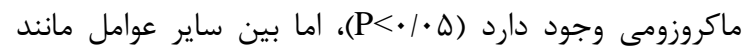

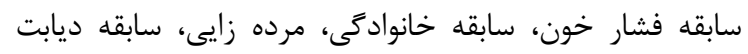

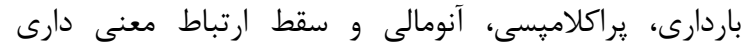

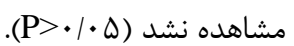

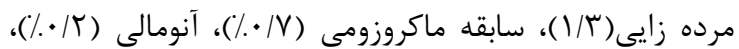

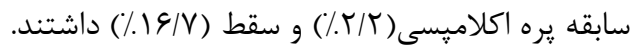

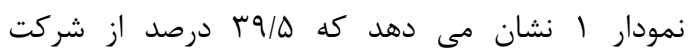
كنندكان ديابت باردارى و لمودران • درصد نيز ديابت آشكار داشتند. نمودار r و r ن نشان مى دهد كه

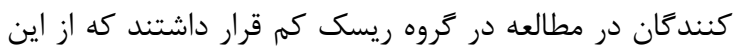

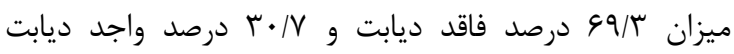
باردارى بوده اند. له/ هو درصد در ريسك متوسط قرار داشتند

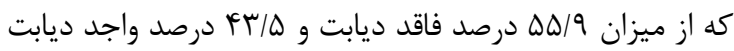

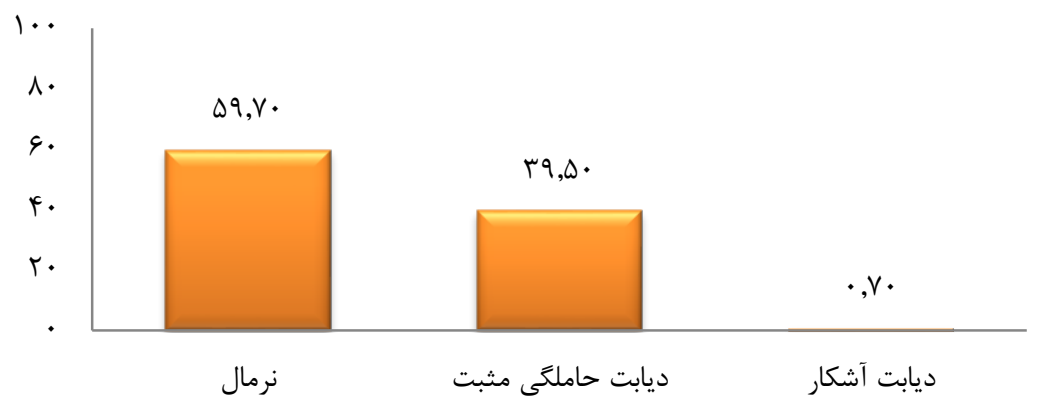

نمودار ا: يزان ابتلا به ديابت باردارى

$\Delta 9,1$.

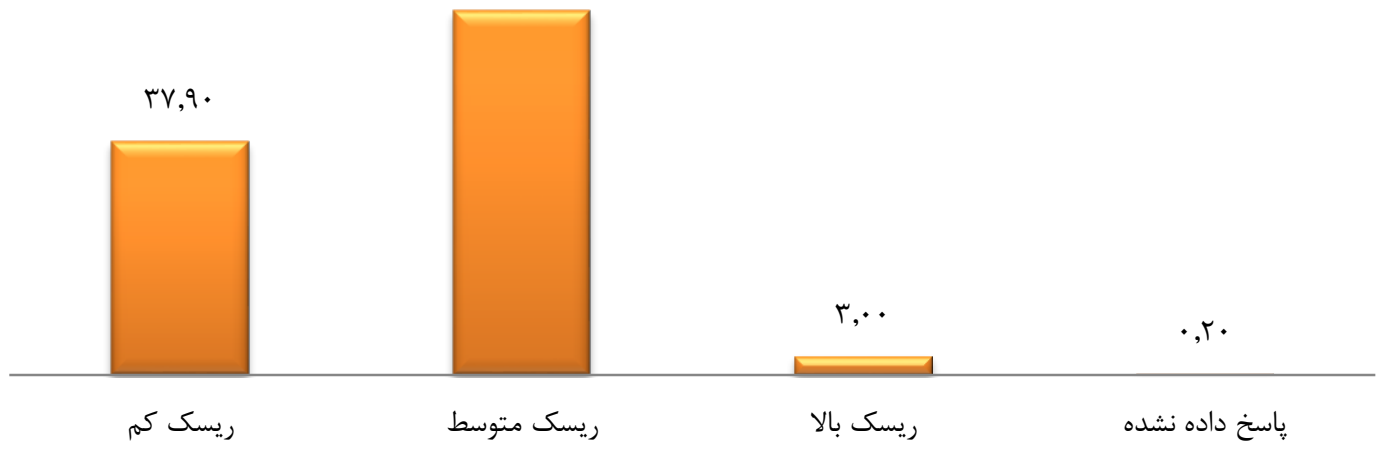

نمودار ب: توزيع فراوانى گروه هاى در معرض خطر ديابت باردارى

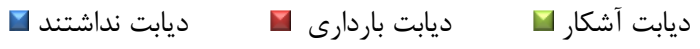

$99, \%$.

$\Delta \Delta, q$.
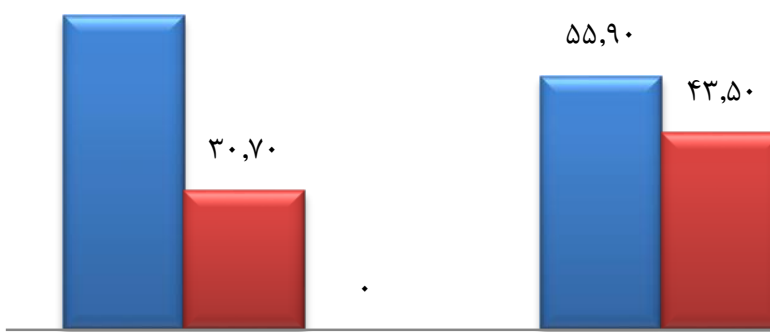

$\Delta \cdot$

ريسك كم

ريسك متوسط

$\cdot, q$.

$\mid r, Q \cdot$

Vo

نمودار بّ: ميزان شيوع ديابت باردارى به تفكيك گروه هاى در معرض خطر 
جدول Y: بررسى ارتباط بين ميزان شيوع ديابت باردارى با عوامل خطر

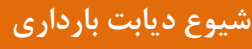

\begin{tabular}{|c|c|c|c|c|c|}
\hline P-Value & منفى (درصد)فراوانى & ديابت حامًالى & ديابت آشكار & طبقه & متغير \\
\hline$\cdot / 4 \cdot r$ & $\begin{array}{l}r \mid Q(\varepsilon \cdot / 1) \\
r(f \cdot)\end{array}$ & $\begin{array}{c}r \cdot \Delta(r q / 1) \\
\varphi(\varphi \cdot)\end{array}$ & $f(\cdot / \Lambda)$ & 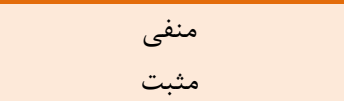 & سابقه فشار خون \\
\hline .1 .90 & $\begin{array}{l}\text { rqY(GY) } \\
\text { rG(FY/G) } \\
I(\Delta \cdot)\end{array}$ & $\begin{array}{l}\operatorname{IVG}(Y V / \mathcal{F}) \\
\operatorname{HF}(\Delta \Delta / V) \\
I(\Delta \cdot)\end{array}$ & $\begin{array}{l}r(\cdot / 9) \\
I(1 / 9) \\
.\end{array}$ & 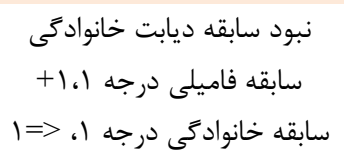 & سابقه خانوادگى ديابت \\
\hline (1/. & $\begin{array}{l}\text { TQY (GT) } \\
\text { TV(FT/Q) }\end{array}$ & $\begin{array}{l}I V G(T V / \Psi) \\
r \Delta(\Delta \Delta / \varepsilon)\end{array}$ & $\begin{array}{l}r(\cdot / 9) \\
I(1 / 9)\end{array}$ & 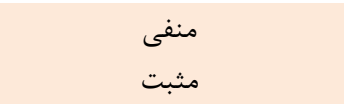 & سلوكز \\
\hline.$/ 1 \mathrm{VV}$ & $\begin{array}{c}r \mid \Lambda(\varepsilon \cdot / 1) \\
I(r \cdot)\end{array}$ & $\begin{array}{c}r \cdot V(r q / 1) \\
r(\Lambda \cdot)\end{array}$ & $f(\cdot / \Lambda)$ & 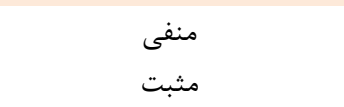 & سابقه ديابت حاملكى قبل \\
\hline$\cdot 11 \cdot 9$ & $\begin{array}{l}M I \mathcal{F}(\Delta q / \varphi) \\
\Delta(Y \backslash / \mathcal{F})\end{array}$ & $\begin{array}{l}r \cdot q(\text { ( } / V) \\
r(r \wedge / q)\end{array}$ & $F(\cdot / \Lambda)$ & مثبت منف & مرده زايى \\
\hline$\cdot 1 \cdot 44$ & $\begin{array}{c}M 19(9 \cdot / r) \\
.\end{array}$ & $\begin{array}{l}r \cdot V(r q / 1) \\
r(1 \cdot \cdots)\end{array}$ & $f(\cdot / \Lambda)$ & مثنى مثت & ماكروزومى \\
\hline$\cdot / \mathrm{VH}$. & $\begin{array}{l}\mu \mid r(\varphi \cdot) \\
\varphi(\Delta \cdot)\end{array}$ & $\begin{array}{c}r \cdot \Delta(\Gamma q / \Gamma) \\
q(\Delta \cdot)\end{array}$ & $f(\cdot / \Lambda)$ & مثبت مثى & يراكلاميسى \\
\hline •/VIT & $\begin{array}{c}\operatorname{MIV}(\Delta q / \varphi) \\
1(1 \cdots)\end{array}$ & $\begin{array}{c}r \|(r q / V) \\
.\end{array}$ & $F(\cdot / \Lambda)$ & 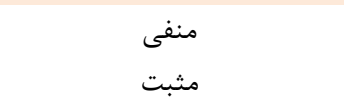 & آنومالى \\
\hline .1 .99 & $\begin{array}{c}r \vee \wedge(G T / Q) \\
F I(F V / I) \\
.\end{array}$ & $\begin{array}{l}1 G F(T G / 9) \\
F \Delta(\Delta \mid / Y) \\
I(1 \cdots)\end{array}$ & $\begin{array}{l}r(\cdot / V) \\
I(1 / 1) \\
.\end{array}$ & $\begin{array}{c}\text { اصلا } \\
1 \\
r\end{array}$ & سقط \\
\hline
\end{tabular}

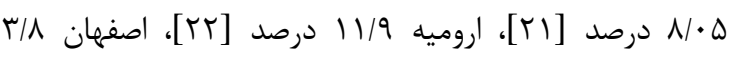

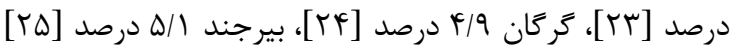

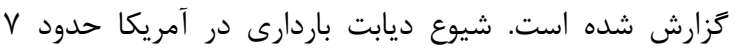

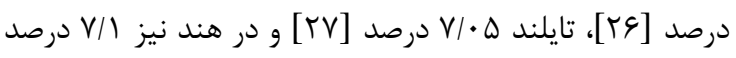

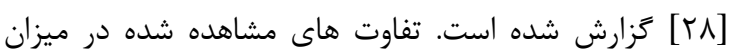

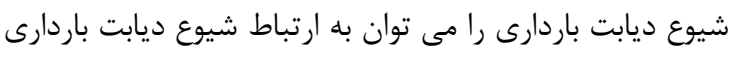

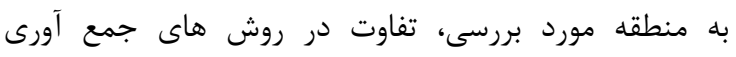

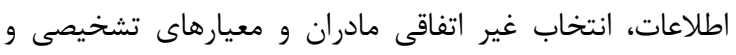

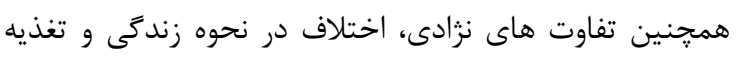
مرتبط دانست. در مطالعه حاضر عوامل خطرساز ديابت باردارى نيز مورد

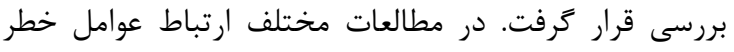

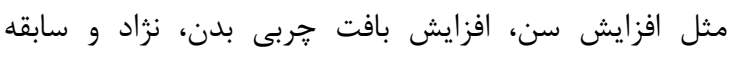

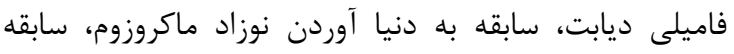
قبلى اختلال تحمل كلوكز در مادر با ديابت باردارى به اثبات رسيده است.

در يروهش حاضر عواملى مانند ماكروزومى و سابقه اختلال

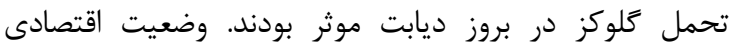

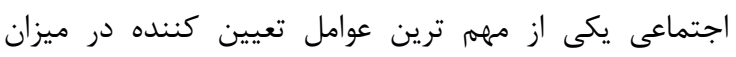

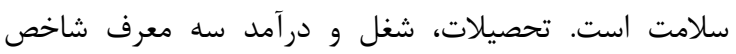

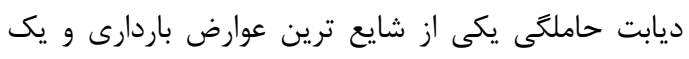

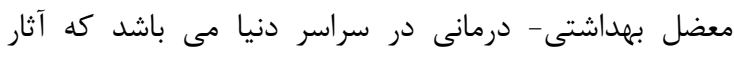

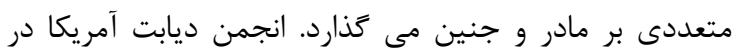

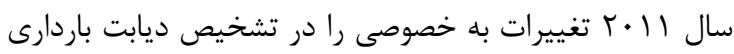

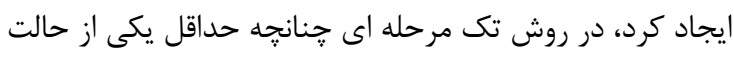
هاى سه گانه وجود داشته باشد، تشخيص ديابت باردارى مرى محرز

مى شود [11]]. در اين مطالعه ميزان شيوع ديابت باردارى در بين زنان

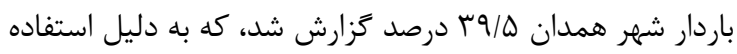

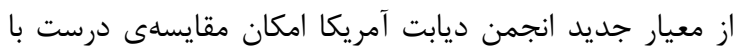

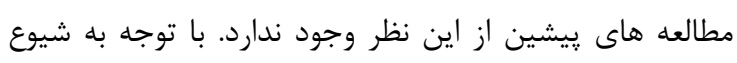

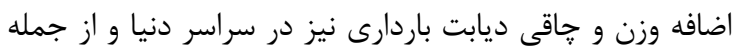

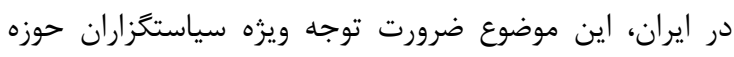

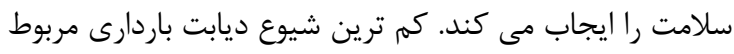

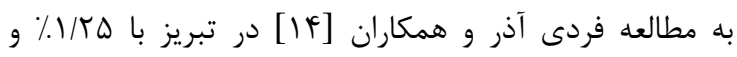

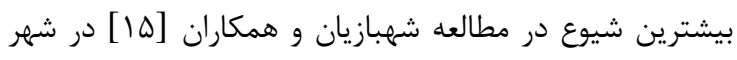

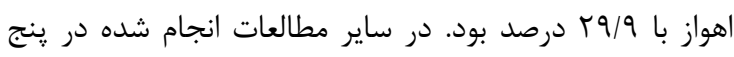

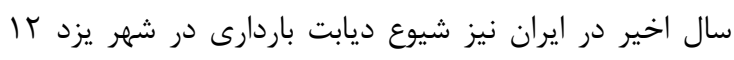

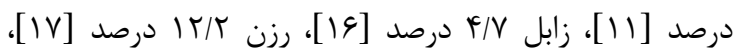

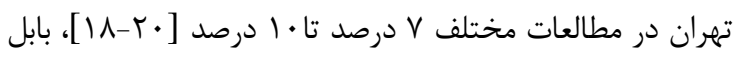




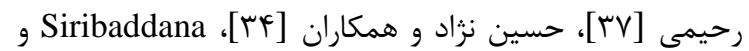

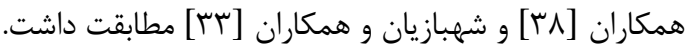

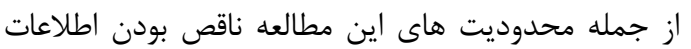

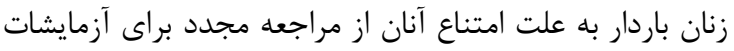

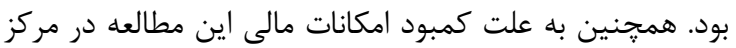
بهداشت اصلى شهر همدان انجام شد كه يِيشنهاد مى كردد

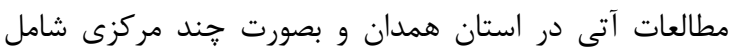
شهرها و روستاهاى اطراف انجام شود.

\section{نتيجه كيرى}

مطالعهى حاضر نشان داد كه در شهر همدان ديابت حاملكى شيوع نسبتا بالايى دارد و اين امر مربوط به جنه جندين

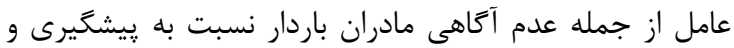

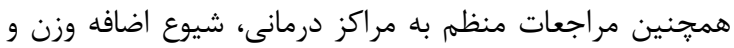

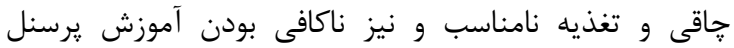

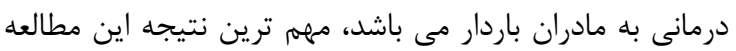

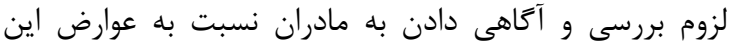

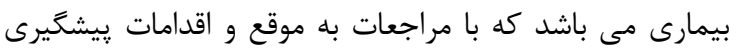

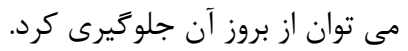

$$
\text { تشعر و قنر قاذى مان }
$$

اين مطالعه با كد طرح

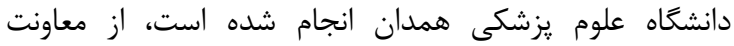
يزوهشى دانشگاه علوم يزشكى همدان و واحد توسعه تحقيقات

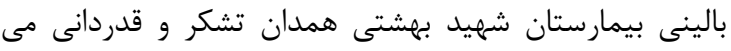

تضاد منافع اين مطالعه براى نويسندگان هيج گَونه تضاد منافعى

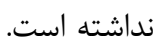

\section{REFERENCES}

1. Crowther C, Hiller J, Moss J, McPhee A, Jeffries W. Robinson JS; Australian Carbohydrate Intolerance Study in Pregnant Women (ACHOIS) Trial Group. Effect of treatment of gestational diabetes mellitus on pregnancy outcomes. N Engl J Med. 2005; 352: 247.

2. Turok DK, Ratcliffe SD, Baxley EG. Management of gestational diabetes mellitus. Am Fam Physician. 2003; 68: $1767-72$

3. WHO. Diabetes. 2017 [updated November 2017; cited 2017 November]; Available from: http://www.who.int/ mediacentre/factsheets/fs312/en/.

4. Ju H, Rumbold AR, Willson KJ, Crowther CA. Borderline gestational diabetes mellitus and pregnancy outcomes. BMC Pregnancy and Childbirth. 2008; 8: 31 .

5. Buckley BS, Harreiter J, Damm P, Corcoy R, Chico A, Simmons D, Vellinga A ,Dunne F. Gestational diabetes mellitus in Europe: prevalence, current screening practice and barriers to screening. A review. Diabet Med. 2012; 29: 844-54.

6. Choudhary N, Rasheed M, Aggarwal V. Prevalence of gestational diabetes mellitus, maternal and neonatal outcomes in a peripheral hospital in North India. Int J Res
اقتصادى اجتماعى در برخى منابع است. خانواده هايى كه در

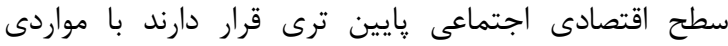

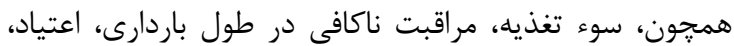
استعمال دخانيات و الكل، باردارى هاى يى در ئى دي، استرس و ...

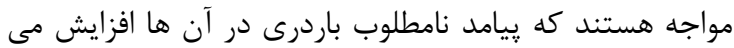

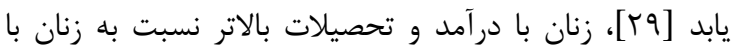

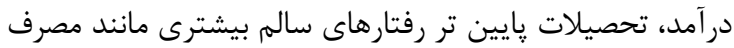

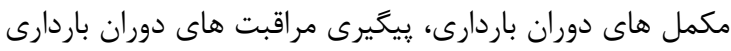

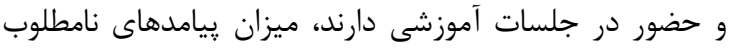

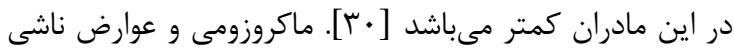

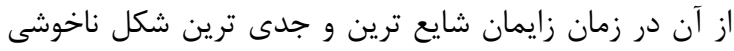

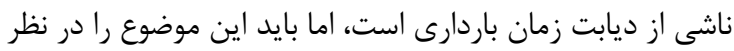
داشت كه ارتباط ضعيفى بين هيير كليسمى مادرى و و وزن زمان زمان

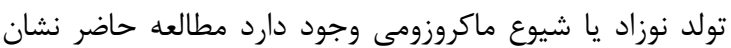

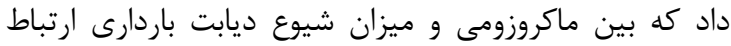

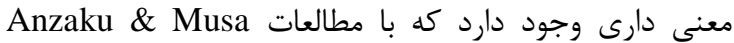

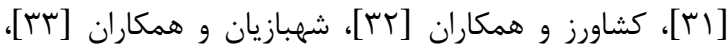

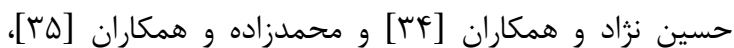

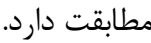
در اين مطالعه سابقه يره اكلامِسى و آنومالى از نظر آمارى دارى

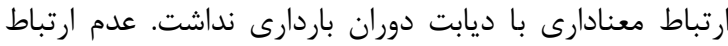

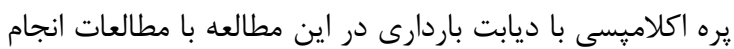
شده توسط كشاورز و همكاران [Tr] مشابه است. اما با مطالعات Anttila دليل تكميل نبودن داده هاى يرونده هاى خانوار در مراكز

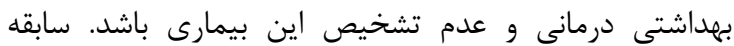

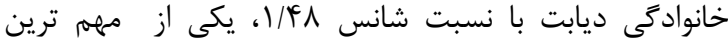

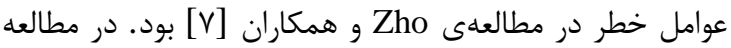

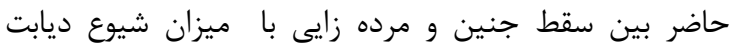

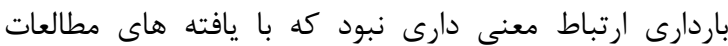

Med Sci. 2017; 5: 2343-5

7. Zhu W-W, Yang H-X, Wang C, Su R-N, Feng H, Kapur A. High Prevalence of Gestational Diabetes Mellitus in Beijing: Effect of Maternal Birth Weight and Other Risk Factors. Chin Med J. 2017; 130: 1019

8. Kahn R. Follow-up report on the diagnosis of diabetes mellitus: the expert committee on the diagnosis and classifications of diabetes mellitus. Diabetes care. 2003; 31: 3162-7.

9. Sayehmiri F, Bakhtiyari S, Darvishi P, Sayehmiri K. Prevalence of Gestational Diabetes Mellitus in Iran: A Systematic Review and Meta-Analysis Study. Iran J Obstet Gynecol Infertil. 2013; 15: 16-23. (Persian)

10. Sharifi N, Dolatian M, Mahmoodi Z, Nasrabadi FM. Gestational diabetes and its relationship with social determinants of health according to world health organization model: Systematic review. Iran $\mathbf{J}$ Obstet Gynecol Infertil. 2017; 19:6-18. (Persian)

11. Vakili M, Pordanjani S, Alipor N, Taheri M, Baeradeh N, Hashemi A. The prevalence of gestational diabetes and associated factors in pregnant women referred to health care centers of Yazd in 2012. J Sabzevar Univ Med Sci. 2015; 
21: 214-24. (Persian)

12. Marathe PH, Gao HX, Close KL. American Diabetes Association standards of medical care in diabetes. J Diabetes. 2017; 9: 320-4.

13. American Diabetes A. Diagnosis and Classification of Diabetes Mellitus. Diabetes Care. 2011; 34.

14. Faridi AZ, Abdollahi-Fard S, Najafipour F, Shahr AE. Evaluation of material complication of overt and gestational diabetes. Iran J Obstet Gynecol Infertil. 2007; 10: 17-24. (Persian)

15. Shahbazian H, Nouhjah S, Shahbazian N, Jahanfar S, Latifi SM, Aleali A, Shahbazian N, Saadati N. Gestational diabetes mellitus in an Iranian pregnant population using IADPSG criteria: incidence, contributing factors and outcomes. Diabetes Metab Syndr. 2016; 10: 242-6. (Persian)

16. Shahdadi H, Mohammadoour HR, Rahnama M, Dindar M, Mastalizadeh H. Study BMI and demographic variables in pregnant women with gestational diabetes. J Diabetes Nurs. 2015; 3:42-50. (Persian)

17. Dorostkar H, Zomorodi Zare N, AlikhaniMahvar A, Goodarzi MT. Prevalence of Gestational Diabetes Mellitus in Different Age Groups in Razan, Iran 2014. J Mazandaran Univ Med Sci. 2015; 25: 74-81. (Persian)

18. Mokhlesi S, Momenzadeh F, Mohebi S, Moghaddam Banaem L. Relationships between Iron and Zinc Serum Levels in early second trimester of pregnancy and gestational diabetes. Alborz Univ Med J. 2014; 3: 127-32. (Persian.

19. Ashrafi M, Sheikhan F, Arabipoor A, Hosseini R, Nourbakhsh F, Zolfaghari Z. Gestational diabetes mellitus risk factors in women with polycystic ovary syndrome (PCOS). Eur J Obstet Gynecol Reprod Biol. 2014; 181: 1959. (Persian)

20. Ashrafi M, Gosili R, Hosseini R, Arabipoor A, Ahmadi J, Chehrazi M. Risk of gestational diabetes mellitus in patients undergoing assisted reproductive techniques. Eur J Obstet Gynecol Reprod Biol. 2014; 176: 149-52. (Persian)

21. Bouzari Z, Yazdani S, Abedi Samakosh M, Mohammadnetaj M, Emamimeybodi S. Prevalence of gestational diabetes and its risk factors in pregnant women referred to Health Centers of Babol, Iran, from september 2010 to march 2012. Iran J Obstet Gynecol Infertil. 2013; 16: 6-13.

22. Manafi M, Khadem-Ansari M. Gestational diabetes mellitus in Iranian women: a rising rate. Acta Endocrinol Buch. 2013; 9: 71-8. (Persian)

23. Goli M, Hemmat AR, Foroughipour A. Risk factors of gestational diabetes mellitus in Iranian pregnant women. Health Syst Res. 2012; 8: 282-9. (Persian)

24. Mohammadzadeh F, Eshghinia S, Vakili MA. The prevalence of gestational diabetes mellitus and its related risk factors in Gorgan, north of Iran. Selective or universal screening test is cost-effective?. Int J Diabetes Dev Ctries. 2015; 35: 225-9. (Persian)
25. Hedayati H, Khazaee T, Mogharrab M, Sharifzadeh GR. Prevalence of gestational diabetes mellitus and overt diabetes in perganant women in Birjand. Modern Care Journal. 2012; 8: 238-44. (Persian)

26. Albrecht SS, Kuklina EV, Bansil P, Jamieson DJ, Whiteman MK, Kourtis AP, Posner SF, Callaghan WM. Diabetes trends among delivery hospitalizations in the US, 19942004. Diabetes care. 2010; 33: 768-73.

27. Chanprapaph P, Sutjarit C. Prevalence of gestational diabetes mellitus (GDM) in women screened by glucose challenge test (GCT) at Maharaj Nakorn Chiang Mai Hospital. J Med Assoc Thai. 2004; 87: 1141-6.

28. Bose $\mathrm{T}$.Incidence of gestational diabetes in general population. J Hum Ecol. 2005; 17: 251-4.

29. ZarbakhshBhari MR, Hoseinian S, Afrooz G, Hooman H. The comparison of many biological characteristics, economical conditions, general health (Mental), of mothers with low and normal birth weight at Guilan province. Payavard Salamat. 2012; 5: 67-78. (Persian)

30. Alizadeh M, Dastgiri S, Taghavi S, Khanlarzadeh E, Khamnian Z, Jafarabadi MA, Hosseini R, Beyrami HJ. The relationship between social determinants of health and pregnancy outcomes: a retrospective cohort study in Tabriz. J Clin Invest \& Gov. 2014; 3: 152-7. (Persian)

31. Anzaku AS, Musa J. Prevalence and associated risk factors for gestational diabetes in Jos, North-central, Nigeria. Arch Gynecol Obstet. 2013; 287: 859-63.

32. Keshavarz M, Cheung NW, Babaee GR, Moghadam HK, Ajami ME, Shariati M. Gestational diabetes in Iran: incidence, risk factors and pregnancy outcomes. Diabetes Res Clin Pract. 2005; 69. 279-86.

33. Shahbazian H, Shahbazian N, Yarahmadi M, Saiedi S. Prevalence of gestational diabetes mellitus in pregnant women referring to gynecology and obstetrics clinics. Jundishapur J Med. 2012; 11: 113-21. (Persian)

34. Hossein-Nezhad A, Maghbooli Z, Vassigh A-R, Larijani B. Prevalence of gestational diabetes mellitus and pregnancy outcomes in Iranian women. Taiwan J Obstet Gynecol. 2007; 46: 236-41. (Persian)

35. Mohammadzadeh F, Mobasheri E, Eshginia S, Kazeminejad V, Vakili M. Prevalence of Gestational Diabetes and its Risk Factors in Pregnant Women in Gorgan, 2011-2011. Iran J Diabetes Metab. 2013; 12: 204-10. (Persian)

36. Anttila L, Karjala K, Penttilä T-A, Ruutiainen K, Ekblad U. Polycystic ovaries in women with gestational diabetes. Obstet Gynecol. 1998; 92: 13-6.

37. Giti R. Prevalence of gestational diabetes mellitus in pregnant women referring to health centers in Ardabil. Res. Sci J Ardabil Univ Med Sci Health Serv. 2004; 4: 32-9.

38. Siribaddana S, Deshabandu R, Rajapakse D, Silva K, Fernando D. The prevalence of gestational diabetes in a Sri Lankan antenatal clinic. Ceylon Med J. 1998; 43: 88-91. 